\title{
L-arginina: suplemento nutricional sin beneficios demostrados en patologías del embarazo
}

\author{
Cristián González Carvallo ${ }^{1}$, Jorge Carvajal C., PhD. ${ }^{2}$ \\ ${ }^{1}$ Programa de Obstetricia y Ginecología, ${ }^{2}$ Unidad de Medicina Materno Fetal. División de Obstetricia y Ginecología, \\ Facultad de Medicina, Pontificia Universidad Católica de Chile.
}

\section{RESUMEN}

Objetivo: Análisis crítico de estudios clínicos randomizados para determinar si el suplemento con L-arginina mejora el resultado perinatal en pacientes embarazadas en riesgo o que presenten patologías como parto prematuro, síndrome hipertensivo del embarazo o restricción de crecimiento fetal intrauterino. Métodos: Se realizó una búsqueda bibliográfica en base de datos Pubmed, Tripdatabase y una multibúsqueda en el Sistema de Bibliotecas de la Pontificia Universidad Católica de Chile con posterior análisis crítico de los estudios seleccionados. Resultados: Se seleccionaron 14 estudios clínicos randomizados; 4 de ellos corresponden a estudios de L-arginina en hipertensión gestacional, 5 estudios de L-arginina en pre-eclampsia, 1 estudio de L-arginina en parto prematuro, 1 estudio de L-arginina en hipertensión crónica y 4 estudios de L-arginina en restricción de crecimiento intrauterino. Solo dos de los estudios son de buena calidad metodológica, pero uno de ellos estudia un resultado intermedio y no clínicamente relevante. El resultado más promisorio muestra que el uso de L-arginina asociada a vitaminas antioxidantes reduce significativamente la incidencia de preeclampsia en mujeres de riesgo (antecedente personal o familiar de preeclampsia). Conclusiones: No se ha demostrado la efectividad de la suplementación antenatal con L-arginina para mejorar el resultado perinatal en embarazadas en riesgo o que presentan patologías como parto prematuro, síndrome hipertensivo del embarazo o restricción de crecimiento fetal. Se requieren estudios de buen diseño que permitan conclusiones definitivas.

\section{PALABRAS CLAVE: L-arginina, preeclampsia, parto prematuro, restricción de crecimiento fetal, hipertensión arterial crónica, síndrome hipertensivo del embarazo}

\section{SUMMARY}

Objective: Critical analysis of randomized clinical trials to determine whether supplementation with L-arginine improves perinatal outcome in pregnant patients at risk or presenting conditions such as preterm labor, hypertensive disorders or intrauterine fetal growth restriction. Methods: We performed a literature search in Pubmed, Tripdatabase and multisearch in the Library System of the Pontifical Catholic University of Chile to further critical analysis of selected studies. Results: We selected 14 randomized trials, 4 of them are for studies of L-arginine in gestational hypertension, 5 studies of L-arginine in preeclampsia, one study of L-arginine in preterm labor, one study of L-arginine in chronic hypertension and 4 studies of L-arginine in intrauterine growth restriction. Only two of the studies were of good methodological quality, but one these studies analyzes an intermediate result that is not clinically relevant. The most promising result shows that the use of L-arginine associated with antioxidant vitamins significantly reduced the incidence of preeclampsia in women at risk (personal or family history of preeclampsia). Conclusions: Is not shown the effectiveness 
of antenatal supplementation with L-arginine to improve perinatal outcome in pregnant women at risk or with pathological conditions such as premature labor, hypertensive disorders and fetal growth restriction. More studies of good design are needed to allow definitive conclusions.

\section{KEY WORDS: L-arginine, preeclampsia, preterm delivery, fetal growth restriction, chronic hypertension, hypertensive disorders}

\section{INTRODUCCION}

El óxido nítrico (NO) es un poderoso vasodilatador, producido por el endotelio vascular (1) que desempeña un rol fundamental en la fisiología reproductiva humana: es una molécula indispensable en placentación, en la fisiología del parto y de la dilatación cervical $(2,3)$, en la génesis de la preeclampsia (4) y restricción del crecimiento intrauterino (5).

La L-arginina es un aminoácido semi-esencial precursor del NO por medio de la enzima óxido nítrico sintetasa (6). Algunos autores han propuesto que en el embarazo existe una deficiencia relativa de L-arginina debido al aumento de la síntesis de NO $(4,7)$. En pacientes que presentan patologías como preeclampsia, hipertensión gestacional y restricción del crecimiento intrauterino, que presentan niveles disminuidos de L-arginina y $\mathrm{NO}$, se cree que el déficit de L-arginina y/o NO juegan un rol en la fisiopatología de estas enfermedades (4). Se postuló que la administración de L-arginina como suplemento nutricional en pacientes con riesgo o que padecen estas patologías (hipertensión, restricción de crecimiento o parto prematuro), podría mejora la biodisponibilidad de NO, disminuyendo la probabilidad de enfermar y/o mejorando los resultados perinatales. Con esta hipótesis se han desarrollado varias investigaciones clínicas que estudian la utilidad de la suplementación con L-arginina durante el embarazo.

El objetivo de este estudio es realizar una revisión de la literatura sobre el uso de la suplementación con L-arginina para mejorar los resultados perinatales en distintas patologías del embarazo, como síndrome hipertensivo del embarazo, parto prematuro y restricción del crecimiento intrauterino.

\section{MATERIAL Y MÉTODOS}

La pregunta de investigación es si la suplementación con L-arginina mejora el resultado perinatal en embarazadas con riesgo o que presentan patologías como parto prematuro, síndrome hipertensivo del embarazo o restricción del crecimiento intrauterino. Para responderla se realizó una búsqueda en las bases de datos PUBMED; Tripdatabase y a través de una multibúsqueda en el Sistema de Bibliotecas de la Pontificia Universidad Católica de Chile. Se utilizaron los términos MESH "Arginine", "Pregnancy, "Preeclampsia", "Premature labor" "Fetal Growth Restriction". La búsqueda se limitó a estudios de los últimos 12 años, en inglés o español, publicados hasta el 10 de junio de 2012, de tipo clínico randomizado que evalúen el uso de Larginina en resultados perinatales.

Con la estrategia de búsqueda se seleccionan 14 estudios clínicos randomizados (8-21); 4 de ellos evalúan uso de L-arginina en hipertensión gestacional, 5 en preeclampsia, 1 en parto prematuro, 1 en hipertensión crónica y 4 en restricción de crecimiento intrauterino. No se incluyó estudios de tipo observacional.

La descripción de los 14 estudios se resume en la Tabla I. Los artículos seleccionados fueron analizados críticamente con las Guías CASPe (Critical Appraisal Skills Programme Español) de Lectura Crítica de la Literatura Médica. Alicante: CASPe; 2005, disponibles en el sitio web www.redcaspe. org. El resumen del análisis crítico de la metodología de los estudios se presenta en la Tabla II.

\section{RESULTADOS}

L-arginina y preeclampsia. Dentro de los 5 trabajos evalúan el efecto de la L-arginina en preeclampsia, existe 1 metodológicamente bueno como se ve en la Tabla II (21), 2 con metodología aceptable $(10,15)$, y 2 con metodología pobre $(12,13)$. A continuación describimos y analizamos metodológicamente cada uno de ellos.

Hladunewich y cols (15), evalúan el uso de la Larginina para mejorar cifras tensionales y reducir el daño glomerular en pacientes puérperas con preeclampsia. Randomizó pacientes con diagnóstico de preeclampsia (embarazo y/o puerperio inmediato), a L-arginina (oral o endovenosa según tolerancia) o placebo. El resultado primario fue la medición de cifras tensionales y la tasa de filtración glomerular a los 10 días del puerperio. No detectó diferencias significativas en ninguno de los dos resultados estudiados. El principal problema de este estudio es la falta de cálculo de tamaño muestral; adicionalmente, incluye pacientes hospitalizados y ambulatorios. No define con precisión los criterios de exclusión; tiene diferencias significativas respecto 
Tabla I

DESCRIPCIÓN DE LOS ESTUDIOS CLÍNICOS RANDOMIZADOS INCLUIDOS

\begin{tabular}{|c|c|c|c|c|}
\hline Autor principal & $\begin{array}{c}\text { Año de } \\
\text { publicación }\end{array}$ & $\begin{array}{l}\mathrm{N}^{\circ} \text { de pacientes } \\
\text { grupo L-arginina }\end{array}$ & $\begin{array}{c}\mathrm{N}^{\circ} \text { de pacientes } \\
\text { grupo placebo }\end{array}$ & $\begin{array}{l}\text { Patología } \\
\text { involucrada }\end{array}$ \\
\hline Neri (8) & 2004 & 15 & 15 & Hipertensión gestacional \\
\hline Sierowzeski (9) & 2004 & 78 & 30 & $\mathrm{RCF}$ \\
\hline Staff (10) & 2004 & 15 & 15 & Preeclampsia \\
\hline Xiao (11) & 2005 & 30 & 36 & $\mathrm{RCF}$ \\
\hline Rytlewski (12) & 2005 & 42 & 41 & Preeclampsia \\
\hline Rytlewski (13) & 2006 & 42 & 41 & Preeclampsia \\
\hline Neri (14) & 2006 & 62 & 61 & Hipertensión gestacional \\
\hline Hladunewich (15) & 2006 & 22 & 23 & Preeclampsia \\
\hline Facchinetti (16) & 2007 & 40 & 40 & Hipertensión gestacional \\
\hline Dera (17) & 2007 & 42 & 27 & Hipertensión gestacional + RCF \\
\hline Rytlewski (18) & 2008 & 37 & 33 & Parto prematuro \\
\hline Winer (19) & 2009 & 21 & 22 & $\mathrm{RCF}$ \\
\hline Neri (20) & 2010 & 40 & 40 & Hipertensión crónica \\
\hline Vadillo-Ortega (21) & 2011 & 228 & 222 & Preeclampsia \\
\hline
\end{tabular}

RCF: Restricción de crecimiento fetal.

Tabla II

ANÁLISIS DE VALIDEZ INTERNA DE LOS ESTUDIOS CLÍNICOS RANDOMIZADOS INCLUIDOS

\begin{tabular}{|c|c|c|c|c|c|c|c|c|}
\hline Autor principal & $\begin{array}{l}\text { Randomi- } \\
\text { zación }\end{array}$ & $\begin{array}{c}\text { Ocultamiento } \\
\text { de } \\
\text { secuencia }\end{array}$ & $\begin{array}{c}\text { Grupos } \\
\text { similares } \\
\text { al inicio }\end{array}$ & $\begin{array}{l}\text { Cálculo } \\
\text { tamaño } \\
\text { muestral }\end{array}$ & Ciego & $\begin{array}{c}\text { Intención } \\
\text { de } \\
\text { tratar }\end{array}$ & Seguimiento & $\begin{array}{c}\text { Cumplimiento } \\
\text { de la } \\
\text { intervención }\end{array}$ \\
\hline Neri (8) & + & ND & + & - & ND & + & $100 \%$ & $100 \%$ \\
\hline Sieroszewski (9) & + & - & - & - & - & - & $59 \%$ & ND. \\
\hline Staff (10) & + & ND & + & + & ND & - & $100 \%$ & $100 \%$ \\
\hline Xiao (11) & ND & ND & + & - & - & ND & $100 \%$ & $100 \%$ \\
\hline Rytlewski (12) & ND & ND & + & - & ND & - & $73 \%$ & $73 \%$ \\
\hline Rytlewski (13) & + & ND & - & - & + & - & $73 \%$ & $73 \%$ \\
\hline Neri (14) & + & + & + & - & + & - & $98 \%$ & $98 \%$ \\
\hline Hladunewich(15) & + & + & + & - & + & + & $100 \%$ & $100 \%$ \\
\hline Facchinetti (16) & + & + & + & - & + & - & $93 \%$ & $90 \%$ \\
\hline Dera (17) & ND & ND & - & - & + & + & $100 \%$ & $100 \%$ \\
\hline Rytlewski (18) & + & ND & + & - & + & - & $64 \%$ & $64 \%$ \\
\hline Winer (19) & + & ND & + & + & + & + & $100 \%$ & $100 \%$ \\
\hline Neri (20) & + & + & + & - & + & + & $100 \%$ & $100 \%$ \\
\hline Vadillo-Ortega (21) & + & + & + & + & + & + & $100 \%$ & $82 \%$ \\
\hline
\end{tabular}

ND: no descrito. 
al momento de la administración de las intervenciones (desde varios días antes hasta varios días después del parto), y diferente vía de administración de la L-arginina (oral o endovenosa).

Vadillo-Ortega y cols (21), evalúan el uso de suplementos alimenticios con L-arginina y vitaminas antioxidantes como prevención de preeclampsia/ eclampsia en pacientes con alto riesgo de presentar esta patología. Alto riesgo de preeclampsia fue definido como: antecedente personal de preeclampsia o pariente de primer grado con antecedente de preeclampsia. Se randomizaron pacientes entre 14 y 32 semanas a 3 grupos; grupo 1 placebo, grupo 2 suplemento alimenticio de L-arginina asociado a vitaminas antioxidantes y grupo 3 vitaminas antioxidantes. Como resultado primario se midió la incidencia de preeclampsia/eclampsia. Este estudio mostró una disminución significativa del riesgo de presentar preeclampsia/eclampsia en el grupo de L-arginina + vitaminas antioxidantes comparado con los otros 2 grupos (vs placebo RRA 0,17; IC $95 \% 0,12$ a 1,21 ; vs vitaminas antioxidantes RRA 0,09; IC95\% 0,05 a 0,14). Como resultado secundario midieron la tasa de partos prematuros totales, siendo significativamente menor en el grupo de L-arginina + vitaminas antioxidantes (RRA 0,09; IC95\% 0,05 a 0,13). Este estudio (21) es el de mejor metodología que encontramos en esta revisión. La principal crítica al diseño es que estudia la reducción global de preeclampsia, sin mostrar resultados potencialmente más relevantes como preeclampsia severa, prematurez extrema, restricción del crecimiento fetal (RCF) severo o morbimortalidad materno-fetal, en los cuales la intervención puede ser de mayor interés. Llama la atención, como fuente de sesgo, la existencia de exclusiones post randomización, disminuyendo el porcentaje de intervención realizado y alejándonos del real efecto de la intervención evaluada. Es importante observar que este estudio no utilizó L-arginina sola, de modo que se muestra el beneficio del uso asociado de L-arginina con vitaminas antioxidantes.

Staff y cols (10), evalúan el efecto de la L-arginina oral en la presión arterial diastólica (PAD) a corto plazo en pacientes con preeclampsia entre 28 a 36 semanas. Randomizó pacientes a uso de $12 \mathrm{~g}$ de L-arginina por 5 días vs placebo. Como resultado primario mide las diferencias en PAD de grupo L-arginina vs control (placebo) después de 2 días de intervención. No muestra diferencias significativas entre ambos grupos $(p=0,4$; incremento de $1 \mathrm{mmHg}$ en grupo L-arginina vs $3 \mathrm{mmHg}$ en grupo placebo). El estudio (10) es de calidad metodológica solo aceptable, con un pequeño número de pacientes (aunque respeta el cálculo del tamaño muestral), no se describe un ocultamiento de secuencia de randomización, no hay ciego adecuado y presenta un análisis por protocolo, excluyendo pacientes perdidas post randomización (sobrevalo- ra el efecto de la intervención). No se describen criterios de exclusión, incluyendo cualquier paciente entre 28 y 36 con preeclampsia, enfermedad renal, cardíaca, preeclampsia severa, malformación fetal, etc. Enfermedades que le dan otro contexto a la preeclampsia y puede responder de distinta manera a la L-arginina.

Rytlewski y cols $(12,13)$, realizaron 2 estudios. El primero (12), es un estudio entre el 2000 al 2003, en el cual se evalúa la presión arterial y la síntesis de NO con el uso prolongado de L-arginina vs placebo en mujeres con preeclampsia. Se randomizaron embarazadas de más de 20 semanas con preeclampsia a recibir L-arginina oral o placebo por 3 semanas, midiendo como resultado primario las cifras tensionales y niveles sanguíneos y urinarios de NO. Luego de 3 semanas de tratamiento se vio diferencia significativa, disminuyendo las cifras tensionales del grupo L-arginina vs placebo (PAM $101,8 \pm 1,5$ vs $108 \pm 1,5 ; p<0,01)$. No hubo diferencias significativas en los niveles de NO plasmático. El segundo estudio (13), con las mismas pacientes del primer estudio, en el cual se evalúa el efecto de la L-arginina en el perfil biofísico, crecimiento fetal, flujo úteroplacentario y resultados neonatales (peso y talla, escore de Apgar al minuto y 5 minutos). Luego de 3 semanas de tratamiento, el grupo de placebo presentaba significativamente mayor cantidad de pacientes con RCF. Durante las 2 primeras semanas de tratamiento, el grupo de L-arginina tuvo incremento significativamente mayor de peso fetal que el placebo, incremento que no se mantuvo las segundas 2 semanas. En el PI de arteria umbilical hubo diferencia significativa a las 3 y 4 semanas respecto a placebo disminuyendo su valor, en arteria cerebral media fue significativo después de 2 semanas, la tercera no y la cuarta también fue significativo, aumentando su valor de PI. El Apgar, en el grupo L-arginina fue significativamente mayor al minuto y cinco minutos comparado con placebo. Los 2 trabajos $(12,13)$ son metodológicamente pobres debido a que en ambos no es descrita una buena randomización, no hay cálculo de tamaño muestral, presentan un análisis por protocolo, y ambos presentan seguimiento y cumplimiento de intervención bajos (73\%).

L-Arginina y restricción del crecimiento intrauterino. Se eligieron 4 estudios en esta categoría, dentro de los cuales 1 es de buena metodología (19), y tres de metodología pobre $(9,11,17)$ (Tabla II).

Winer y cols (19), evalúan el uso de L-arginina oral vs placebo en embarazadas con diagnóstico de RCF severo y compara como medidas principales de resultado: peso de nacimiento y morbilidad neonatal (Apgar a los 5 minutos, estado ácido base y requerimiento de $\mathrm{O} 2$ ). Se define $\mathrm{RCF}$ severo como circunferencia abdominal menor al percentil 3 y con alteración en el doppler de arterias uterinas (notch uterino o PI elevado). No hubo diferencias significa- 
tivas en ninguno de los resultados medidos entre los 2 grupos evaluados $(1042 \pm 476 \mathrm{~g}$ vs $1068 \pm$ $452 \mathrm{~g}, \mathrm{p}=0,66)$. Este estudio es metodológicamente bueno (19), como se muestra en la Tabla II. Sin embargo, pese a ser metodológicamente correcto, dudamos de su concepción científica si consideramos que es poco probable el éxito terapéutico en pacientes con pronóstico tan malo (RCF severa) y en quienes probablemente la noxa comienza en las primeras semanas de gestación.

Dera y cols (17), evalúan L-arginina oral vs placebo, respecto al resultado neonatal en fetos mayores a 24 semanas con RCF (menor al percentil 10) e hipertensión gestacional. Los resultados neonatales evaluados fueron: peso de nacimiento, tipo de parto, edad gestacional del parto, estado ácidobase, Apgar al minuto y 5 minutos, incidencia de infecciones, hemorragia intracerebral y síndrome de distrés respiratorio. El grupo L-arginina presentó mejora significativa en peso al nacer, edad gestacional al parto, Apgar al minuto y cinco minutos, $\mathrm{pH}$ de cordón y menor tasa de cesáreas. El resultado neonatal neonatal fue significativamente mejor en $\mathrm{pH}$ venoso, menor hemorragia intracerebral en todos sus grados y menor porcentaje de síndrome de distrés respiratorio. Estudio de metodología pobre (Tabla II), que a pesar de indicar que es un estudio clínico randomizado, esto no está descrito en su metodología; además, no presenta cálculo de tamaño muestral y los grupos de tratamiento o placebo son de diferente tamaño.

Sieroszewski y cols (9), evalúan el efecto en incremento de peso fetal de la suplementación con L-arginina oral por 20 días vs no tratamiento, en pacientes con RCF (percentil <10). Como resultado primario se midió el peso fetal por ecografía a los 20 días de tratamiento. El resultado secundario que vale la pena mencionar es el peso del RN. Los resultados de este estudio mostraron un incremento significativamente mayor de peso en el grupo Larginina comparado con el control $(648 \pm 94 \mathrm{~g}$ vs $404 \pm 82 \mathrm{~g}$, respectivamente, $\mathrm{p}=0,012)$. También se pudo ver que hubo diferencia estadísticamente significativa en relación a los pesos de los recién nacidos $(2823 \mathrm{~g} \pm 85 \mathrm{~g}$ en grupo L-arginina vs 2495 $g \pm 147$ control, $p=0,027)$. Este estudio presenta una metodología pobre (Tabla II); no existe cálculo del tamaño muestral, no se especifica la severidad de la RCF en los fetos ingresados al estudio, existe poca explicación de los métodos, la randomización no fue bien explicitada y no se usó placebo en el grupo control.

Xiao y cols (11), evalúan el uso de L-arginina endovenosa vs placebo, por 7 días, en el tratamiento de RCF (percentil $<10$ ). Como resultado primario se midieron niveles de NO materno y peso del RN. También se compararon con un grupo control de embarazadas sanas. Se observó que hubo diferencias significativas de aumento de niveles de NO materno al final del embarazo en el grupo de L-arginina (placebo 43,49 $\pm 20,27 \mathrm{umol} / \mathrm{L}$ vs L-arginina $58,42 \pm 23,12 \mathrm{umol} / \mathrm{L}, \mathrm{p}<0,01)$. El grupo placebo presentó disminución significativa de los niveles de NO comparado con el grupo control sano (placebo $16,95 \pm 11,19$ vs control: 41,01 $\pm 12,49, p<0,01)$. Respecto al peso del RN el grupo de L-arginina presentó un aumento significativo comparado con placebo $(2971,7 \pm 350,1$ vs $2794,4 \pm 339,1, p<0,05)$, sin mayores diferencias en edad gestacional al parto. Este estudio es también metodológicamente pobre como se ve en la Tabla II. La principal crítica es que el resultado primario es un evento intermedio, de poca relevancia clínica (niveles de NO sanguíneo) sin medir el resultado que realmente se pretende estudiar (incidencia de RCF). Adicionalmente no se indica el método de randomización y no hay cálculo del tamaño muestral

L-arginina e hipertensión gestacional. Dentro de los 4 trabajos en que se evalúa la L-arginina en hipertensión gestacional, 1 es de calidad metodológica aceptable (14) y 3 de metodología pobre $(8,16,17)$, este último ya comentado en la sección previa (Tabla II).

Neri y cols (14), evalúan el efecto de la L-arginina endovenosa vs placebo en embarazadas con hipertensión gestacional. Como resultado primario mide las cifras tensionales; después de 4 días de tratamiento la L-arginina presenta eficacia significativa en bajar las cifras tensionales (sistólicas y diastólicas) respecto a placebo (sistólica $-5,5 \%$ vs $-2,2 \%, p<0,001$; diastólica $-7,9 \%$ vs $-4,4 \%$, $\mathrm{p}<0,001)$. Este estudio no presenta cálculo de tamaño muestral y el análisis no es por intención de tratar, lo que podría sobreestimar la magnitud de la intervención. El estudio carece de relevancia clínica al no evaluar resultados perinatales relevantes como RCF o parto prematuro a consecuencia de la HTA. Además, la intervención es breve, lo que no permite conclusiones respecto del uso sostenido de L-arginina.

Facchinetti y cols (16), investigan el efecto de la suplementación con L-arginina (carga de L-arginina ev por 5 días, luego oral hasta completar 2 semanas) comparado con placebo en relación a cifras tensionales y eventos clínicos como latencia al parto, edad gestacional al parto, tipo de parto y peso al nacer. Este estudio incluyó también pacientes con HTA con o sin preeclampsia moderada. Los resultados muestran disminución significativa en cifras tensionales en el grupo de L-arginina (sistólicas $133,2 \pm 10,1$ vs $138,6 \pm 9,1, p=0,02$; diastólicas $81,8 \pm 8,1$ vs $86,7 \pm 8,8, p=0,02)$, y latencia al parto $(31,7 \pm 25,2$ días vs $19,5 \pm 16,9 ; p=0,08)$. Este trabajo no muestra cálculo de tamaño muestral y el análisis no es según intención de tratar. Además, compara grupos pacientes distintos, preeclampsia e hipertensión gestacional, que se comportan de forma distinta y presentan pronósticos diferentes, 
lo que podría alterar el resultado final. Entre los resultados el único de relevancia clínica es la latencia al parto.

Neri y cols (8), evalúan el efecto de la infusión con L-arginina (20 g en infusión de suero fisiológico) vs placebo en los registros basales no estresantes (RBNE) y las cifras tensionales en embarazas con hipertensión gestacional. Después de 40 minutos de infusión se les realizó un RBNE y medición de cifras tensionales. Respecto a la medición de un RBNE no hubo diferencias significativas en ambos grupos. Si lo hubo en las cifras tensionales medidas, las cuales fueron significativamente menores en el grupo de L-arginina (no hay datos de cuanto disminuyó la presión arterial). Este estudio no presenta cálculo de tamaño muestral, el ciego no es claramente descrito en los métodos, y los resultados medidos no tienen significado clínico en relación a la patología estudiada.

L-arginina y parto prematuro. Existe un solo trabajo que evalúa el efecto de la L-arginina en parto prematuro, y es de pobre calidad metodológica (18) (Tabla II). Este estudio observa el efecto que tiene la L-arginina oral, en la circulación uteroplacentaria de fetos de embarazadas con síntomas de parto prematuro. El resultado primario de este estudio fue cambios en los PI de arteria umbilical y cerebral media. Se les hizo medición doppler de arteria umbilical y ACM al inicio, luego a las 2 semanas y posteriormente cada 1 semana hasta el parto. Lo que se observó fue que a las 2 semanas el PI de AU era significativamente menor en el grupo de L-arginina $(0,95 \pm 0,23$ vs $1,07 \pm 0,14, p=0,011)$. Después de la tercera semana el PI de ACM aumentó significativamente en el grupo de L-arginina $(2,18 \pm 0,56$ vs $1,02 \pm(0,15, p=0,018)$, manteniéndose ambos resultados hasta el parto. Este estudio no presenta cálculo de tamaño muestral y el seguimiento es bajo, pudiendo perderse pacientes con resultados negativos cambiando significativamente los resultados globales. Los pacientes incluidos representan un grupo disperso de situaciones clínicas, pueden entrar al estudio cualquier tipo de parto prematuro. La principal crítica es que el resultado medido, doppler umbilical y ACM, es clínicamente irrelevante (el estudio pretende demostrar reducción del parto prematuro). Este estudio presenta objetivos poco claros y no muestra datos relevantes como el tipo de tocolisis usada por grupo, o la duración de este, datos que podrían alterar los resultados.

L-arginina en hipertensión crónica. Se encontró un estudio (20), metodológicamente pobre, que analizó el uso de L-arginina en embarazadas con hipertensión crónica. El estudio evalúa el efecto de L-arginina oral respecto a la presión arterial, complicaciones maternas y neonatales. El resultado primario fue el descenso de cifras tensionales luego de 10-12 semanas de tratamiento. Resultados secundarios fueron uso de hipotensores, parto antes de las 37 y 34 semanas, peso al nacer menor a 2500 o 1500 gramos o menor al percentil 10 e ingreso a UCl neonatal. A las 10-12 semanas de tratamiento se midió el promedio de cifras tensionales. El resto de los parámetros se midieron al momento del parto. No se observó diferencias significativas respecto al resultado primario (sistólica 133,2 \pm 8,8 vs $133,7 \pm 13,2$; diastólica $83,5 \pm 6,3$ vs $81,3 \pm$ $9,8)$. Respecto a los resultados secundarios fueron estadísticamente significativos las diferencias en el uso de hipotensores ( $24 \%$ vs $45 \%, p<0,05$ ), edad gestacional al parto $(38,2 \pm 2,7$ vs $36,6 \pm 3,8$ semanas; $p<0,05)$ y pesos de nacimiento $(3094 \pm 719$ $g$ vs $2836 \pm 946 \mathrm{~g} ; \mathrm{p}<0,05)$, siendo todos favorables al grupo de L-arginina. El estudio no presenta cálculo de tamaño muestral, impidiendo saber si tiene poder suficiente para demostrar la hipótesis estudiada. Además, el resultado primario es intermedio, clínicamente poco relevante, dejando los resultados clínicamente relevantes como medidas secundarias. Más mujeres en el grupo placebo requirieron uso de hipotensores, lo que puede sesgar los resultados (cointervención). Se excluyeron pacientes con HTA severa lo que disminuye la aplicabilidad de los resultados.

\section{DISCUSIÓN}

Con la estrategia de búsqueda se seleccionan 14 investigaciones clínicas randomizadas que evalúan los beneficios de la suplementación con L-arginina durante el embarazo; 5 de ellos evalúan el efecto de L-arginina en preeclampsia, 4 estudios en restricción de crecimiento intrauterino, 4 estudios en hipertensión gestacional, 1 estudio en parto prematuro y 1 estudio en hipertensión crónica.

Los estudios que investigan L-arginina en preeclampsia, evalúan mayoritariamente resultados intermedios $(10,12,15)$ como disminución de presión arterial o niveles de NO sanguíneo o urinario, que no son clínicamente relevantes y no cambian el pronóstico o manejo de la enfermedad. Solamente 2 estudios $(13,21)$ evalúan resultados de relevancia clínica, como tasa de preeclampsia, o resultados neonatales. Solo uno de ellos (21) es metodológicamente bueno, con resultados alentadores e interesantes al mostrar que L-arginina + vitaminas antioxidantes reducen significativamente la incidencia de preeclampsia en embarazadas de alto riesgo. Se usa como grupo de riesgo a mujeres con antecedentes de preeclampsia y a quienes tiene un antecedente familiar de preeclampsia; por lo que sus resultados no pueden extrapolarse a población de bajo riesgo o a otros grupos de alto riesgo (ej. HTA crónica, patología renal, etc.). Estimamos que este único resultado no permite el uso generalizado de L-arginina + vitaminas antioxidantes para prevención primaria o secundaria de preeclampsia, sin embargo, abre las puertas a nuevos estudios clí- 
nicos randomizados bien diseñados en población de alto y/o bajo riesgo como prevención de preeclampsia.

Los trabajos que evalúan el efecto de la L-arginina en RCF, si bien presentan resultados clínicamente relevantes $(11,17,19)$, son estudios metodológicamente pobres, haciendo sus resultados poco válidos. El único estudio metodológicamente bueno (19), concluyó que la L-arginina no mejoraba el resultado perinatal en RCF severa de origen uteroplacentario. Sin embargo, estimamos que la intervención fue aplicada tardíamente en el embarazo, impidiendo algún potencial beneficio. Postulamos la necesidad de una investigación clínica que evalúe el uso de L-arginina en prevención de RCF en pacientes de alto riesgo, pero iniciando la intervención precozmente en el embarazo.

Dos de los estudios que evaluaron L-arginina en hipertensión gestacional muestran resultados con relevancia clínica y pronóstica $(16,17)$, sin embargo su metodología es pobre. Los otros 2 solo estudios muestran resultados intermedios $(8,14)$, impidiendo conclusiones de relevancia en la práctica clínica.

Solo un estudio evaluó el uso de L-arginina en parto prematuro (18) y uno en HTA crónica (20). Ambos son de metodología pobre y cometen el error de mostrar resultados intermedios (doppler fetal y descenso de cifras tensionales, respectivamente) sin estudiar efectos clínicamente significativos como tasa de parto prematuro o aparición de preeclampsia, por ejemplo.

En resumen existen pocos estudios clínicos randomizados que evalúen el efecto de la suplementación con L-arginina en el resultado perinatal del embarazo, siendo la mayoría de metodologías pobres y destinadas a mostrar efectos intermedios y no resultados clínicamente significativos.

No existen estudios que investiguen el efecto de los metabolitos del catabolismo de la L-arginina en el embarazo, como para asegurar que es una droga inocua con efectos puramente positivos en las pacientes que utilizan la L-arginina como suplemento durante el embarazo.

\section{CONCLUSIÓN}

La información científica actual no hace recomendable la suplementación con L-arginina durante el embarazo para mejorar el resultado perinatal en ninguno de los escenarios clínicos evaluados. Es indispensable la realización de investigaciones clínicas randomizadas de buen diseño, destinadas a estudiar el efecto de la suplementación con L-arginina en resultados clínicamente relevantes, como disminución de preeclampsia severa o prematurez, o prevención de restricción de crecimiento intrauterino.

\section{REFERENCIAS}

1. Wu G, Morris SM Jr. Arginine metabolism: nitric oxide and beyond. Biochem J 1998;336:1-17.

2. Norman JE, Cameron IT. Nitric oxide in the human uterus. Rev Reprod 1996;1:61-8.

3. Maul H, Longo M, Saade GR, Garfield RE. Nitric oxide and its role during pregnancy: from ovulation to delivery. Curr Pharm Des 2003;9:359-80.

4. Morris N, Eaton BM. Nitric oxide, the endothelium, pregnancy and pre-eclampsia. Br J Obstet Gynaecol 1996;103:4-15.

5. Wu G, Bazer FW, Cudd TA, Meininger CJ, Spencer TE. Maternal nutrition and fetal development. J Nutr 2004;134:2169-72.

6. Lowenstein CJ, Dinerman JL, Snyder SH. Nitric oxide: A physiologic messenger. Ann Intern Med 1994;120:227-37.

7. Conrad KP, Joffe GM, Kruszyna H, Kruszina R, Rochelle LG, Smith RP, et al. Identification of increased nitric oxide biosynthesis during pregnancy in rats. FASEB J 1993;7:566-71.

8. Neri I, Blasi I, Facchinetti F. Effects of acute L-arginine infusion on non-stress test in hypertensive pregnant women. J Matern Fetal Neonatal Med 2004;16:23-6.

9. Sieroszewski P, Suzin J, Karowicz-Bilińska A. Ultrasound evaluation of intrauterine growth restriction therapy by a nitric oxide donor (L-arginine). J Matern Fetal Neonatal Med 2004;15:363-6

10. Staff AC, Berge L, Haugen G, Lorenzen B, Mikkelsen $B$, Henriksen T. Dietary supplementation with L-arginine or placebo in women with pre-eclampsia. Acta Obstet Gynecol Scand 2004;83:103-7.

11. Xiao XM, Li LP. L-arginine treatment for asymmetric fetal growth restriction. Int J Gynecol Obstet 2005;88:15-8.

12. Rytlewski K, Olszanecki R, Korbut R, Zdebski Z. Effects of prolonged oral supplementation with L-arginine on blood pressure and nitric oxide synthesis in preeclampsia. Eur J Clin Invest 2005;35:32-7.

13. Rytlewski K, Olszanecki R, Lauterbach R, Grzyb A, Basta A. Effects of oral L-arginine on the foetal condition and neonatal outcome in preeclampsia: a preliminary report. Basic Clin Pharmacol Toxicol 2006;99:146-52.

14. Neri I, Jasonni VM, Gori GF, Blasi I, Facchinetti F, for the Arginine Study Group. Effect of L-arginine on blood pressure in pregnancy-induced hypertension: a randomized placebo-controlled trial. J Matern Fetal Neonatal Med 2006;19:277-81

15. Hladunewich MA, Derby GC, Lafayette RA, Blouch $\mathrm{KL}$, Druzin ML, Myers BD. Effect of L-arginine therapy on the glomerular injury of preeclampsia: a randomized controlled trial. Obstet Gynecol 2006;107:886-95.

16. Facchinetti $F$, Saade GR, Neri I, Pizzi C, Longo M, Volpe A. L-arginine supplementation in patients with gestational hypertension: a pilot study. Hypertens Pregnancy 2007;26:121-30. 
17. Dera A, Ropacka M, Kowalska J, Markwitz W, Nycz P, Breborowicz $\mathrm{GH}$. The effect of L-arginine treatment on the neonatal outcome from pregnancies complicated by intrauterine growth restriction and gestational hypertension. Arch Perinat Med 2007;13:35-9.

18. Rytlewski K, Olszanecki R, Lauterbach R, Grzyb A, Kiec-Wilk B, Dembinska-Kiec A, Basta A. Effects of oral L-arginine on the pulsatility indices of umbilical artery and middle cerebral artery in preterm labor. Eur $\mathrm{J}$ Obstet Gynecol Reprod Biol 2008;138:23-8.

19. Winer N, Branger B, Azria E, Tsatsaris V, Philippe HJ, Rozé JC, et al. L-arginine treatment for severe vascular fetal intrauterine growth restriction: a randomized double-bind controlled trial. Clin Nutr 2009;28:243-8.

20. Neri I, Monari F, Sgarbi L, Berardi A, Masellis G, Facchinetti $F$. L-arginine supplementation in women with chronic hypertension: impact on blood pressure and maternal and neonatal complications. J Matern Fetal Neonatal Med 2010;23:1456-60.

21. Vadillo-Ortega F, Perichart-Perera O, Espino S, AvilaVergara MA, Ibarra I, Ahued R, Godines M, Parry S, Macones G, Strauss JF. Effect of supplementation during pregnancy with L-arginine and antioxidant vitamins in medical food on pre-eclampsia in high risk population: randomised controlled trial. BMJ 2011;19;342:d2901. 